\title{
The Effects Playing Pokémon GO has on Physical Activity -A Systematic Literature Review
}

\author{
Samuli Laato \\ University of Turku \\ sadala@utu.fi
}

\author{
Sonja M Hyrynsalmi \\ University of Turku \\ smnyla@utu.fi
}

\author{
Sampsa Rauti \\ University of Turku \\ sjprau@utu.fi
}

\author{
Erkki Sutinen \\ University of Turku \\ erkki.sutinen@utu.fi
}

\begin{abstract}
It is rare for a game to become such a phenomenon that almost 300 scientific papers are published about it in a span of three years. However, that is the case with the location-based game Pokémon GO. The game has been praised as a solution to the global inactivity crisis, but also criticized, for example, for only increasing physical activity in the short term. In order to gather together the findings of previous studies, a systematic literature review was conducted. Initially, 277 scientific articles which contained the term "Pokémon GO" in the title or the abstract were discovered. After categorizing the articles and removing those which did not focus on the effects Pokémon GO has on physical activity, 20 empirical studies remained. A majority (60\%) of the studies showed an increase in the number of daily steps taken, whereas $30 \%$ of studies reported only short term improvements which diminished soon after. The beneficial effects were found to manifest more strongly on physically inactive individuals and were linked to playing retention. Furthermore, the results revealed that (1) most data used in studies is from 2016, after which the game has drastically changed and (2) longitudinal studies spanning over the course of a year are missing.
\end{abstract}

\section{Introduction}

The aim of this study is to investigate the effects of playing the location-based game (LBG) Pokémon $\mathrm{GO}$ on physical activity via synthesizing findings from previous studies. Pokémon GO is a smartphone application which requires a data connection and a GPS-signal to work. The goal of the game is to move around in the real world collecting different Pokémon, and since its launch in summer 2016, the game has maintained a strong player base despite the initial enthusiasm dying out [1]. As of spring 2019, it is still by far the most popular LBG on both iOS and Android platforms [2]. Besides the direct influence the game has on its player, the actions players take in Pokémon
GO are not confined within the game, but influence reality as the game is played in the real world [3].The novelty and popularity of the game has also sparked the interest of academia, with, for example, Scopus currently as of 22th of August having indexed 300 scientific publications where the term "Pokémon GO" appears in the title or the abstract. Pokemon GO has been studied by multiple disciplines such as psychology [4], system sciences [5], law [6], business [7] digital media [8], health $[9,10]$, geography [11], education $[12,2]$ and computer science $[13,14]$ among others.

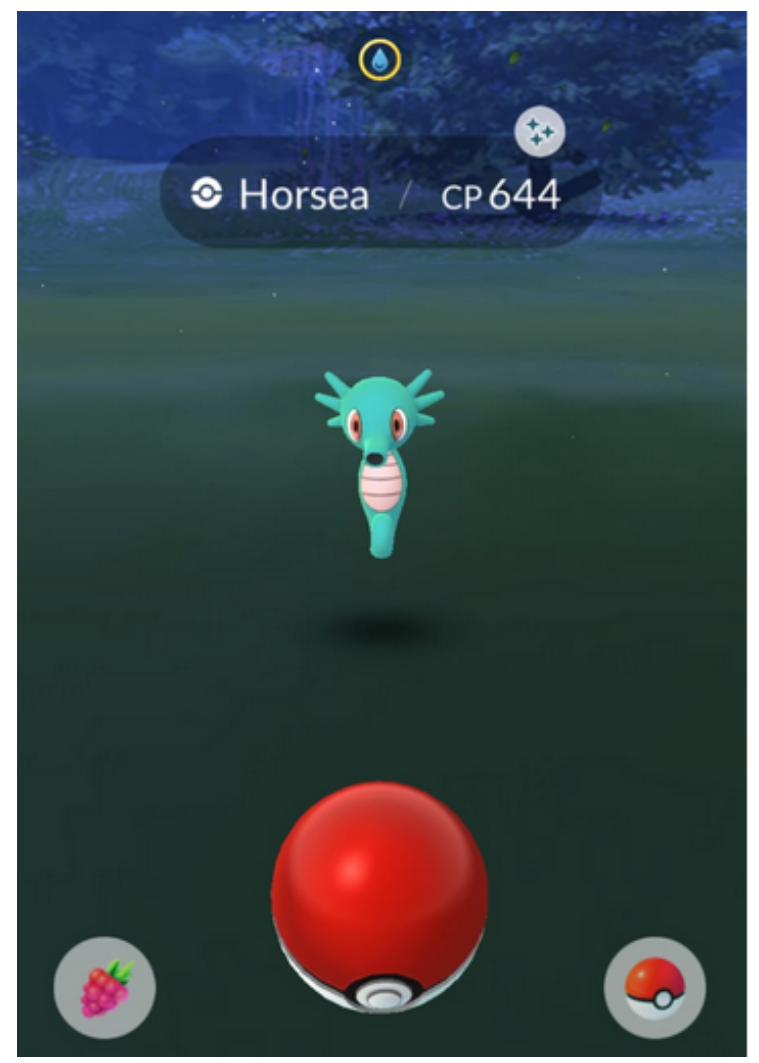

Figure 1. A screenshot from the main Pokeémon capture minigame of Pokémon GO

Pokémon GO can be regarded as a health behavior 
change support system (HBCSS) [15, 16], as, for example, a study observing Pokémon GO players for a period of 10 months showed a substantial increase in physical activity [17]. However, the game is not a traditional health application in the sense that it is designed for entertainment [18]. The main user interface of the game shows a real map, based on OpenStreetMaps, which is skinned to match the graphics of the game. The player character appears on this map, and its location matches the real world location of the player. The main gameplay consists of navigating to points of interest (PoIs) which appear on the map when the player is in their proximity [19]. Upon reaching a PoI, the game app allows the player to interact with it. Pokémon GO currently has three types of PoIs: pokéstops, gyms and pokémon. The first two correspond to real world objects, for example, libraries, churches and statues, whereas pokémon spawns are seemingly more random, and not tied to any specific real world objects [13]. When clicking a Pokémon spawn, a catch minigame shown in Figure 1 emerges. After completing the minigame, the player is returned to the main map interface.

In addition to navigating to PoIs, Pokémon GO includes eggs which are hatched by walking a certain distance with them in an incubator and a buddy pokémon which also needs to be walked with. The main mechanisms the game provides for exercise can thus be summarized as (1) moving around to find PoIs, (2) navigating to PoIs and (3) walking to hatch eggs and to gain buddy candy. All three mechanics consists of moving, and despite Pokémon GO restraining the speed limit of moving to roughly $30 \mathrm{~km} / \mathrm{h}$, there are no further limitations to how the player moves. Thus, it can be either walking, running, cycling, driving etc. [20]. Unlike many other exergames [21], Pokémon GO does not specifically tell the user to exercise, but simply provides gameplay which subtly encourages it. However, recent updates to Pokémon GO have added game mechanics which directly reward walking. The game now provides players feedback of how much they walk per week, and reward them for exercise [22]. These kinds of changes have brought the game closer to typical HBCSSs [16, 23].

\section{Background}

\subsection{The Predecessors of Pokémon GO}

Pokémon GO is not the first exergame [24] or HBCSS [16] in the Pokémon franchise. The remakes of the 2nd Generation Pokémon games, Heart Gold and Soul Silver, were published in autumn 2009 in Japan and then later in spring 2010 in the rest of the world for the handheld device NintendoDS [25]. Even though the games themselves were not influenced by the movement or the location of the player, the games shipped with a special device called Pokéwalker, which was able to count the users' steps via a simple accelerometer [26]. The players could load a pokémon into the Pokéwalker, and level it up by walking around, which resulted in some scholars referring to Heart Gold and Soul Silver as the first exergame (game for exercise) in the Pokémon franchise [27]. Compared to Pokémon GO, the Pokéwalker of Heart Gold and Soul Silver was a simple device. It had very limited interaction and feedback capabilities and was not a standalone game. Regardless it managed to utilize the digital world of Pokémon in motivating players to go out and exercise. [26]

A pedometer like Pokéwalker has not been the only attempt prior to Pokémon GO Nintendo made to encourage players to being physically active. For example, the Nintendo handheld console, Nintendo3DS, which was released in early 2011, came with a special software called StreetPass, which detected nearby fellow 3DS users and automatically sent a greeting to them. 3DS users would benefit from meeting nearby people by gaining puzzle pieces and credits for various minigame [28]. Studies found that the StreetPass feature managed to enrich the gaming experience and increased the sense of belonging to a community and the majority of 3DS users reported that they take the device with them when travelling in order to get StreetPass hits [29]. These findings indicate that social in-game events can also serve as a motivator for players to go out and exercise. The design of Pokémon GO currently incorporates several social elements: (1) raids, where up to 20 players team up to defeat a strong foe [30], (2) trading, where two players get together to trade pokémon (3) PvP battles, where two players can face each other in synchronous combat and (4) standard gym battles, where players from the same team can work together to conquer special PoIs called gyms [22].

In fact, encouraging people to get together has been a part of the main series Pokémon games ever since Pokémon Red and Green were first published in 1996 in Japan [25]. With each generation in the main series of Pokémon games, up to at least generation 4, new ways encouraging players to meet in the real world to play together have been added:

- Red and Blue featured link cable trading

- Silver and Gold added mystery gifts

- Ruby and Sapphire added Secret Bases which 
could be exchanged between players when interacting in the real world

- Diamond and Pearl added odd keystone quests in an underground world which could be played together

The social elements in these games serve at least two purposes. Firstly, players are encouraged to get together and travel to meet each other. Secondly, the social connections increase a sense of belonging and provide further motivation to play [31]. Furthermore, the world of Pokémon has reportedly inspired the imagination of children as it has been incorporated in their playground playing [32]. Social elements in exergames have been found to increasing exercise and helping players become more active in general $[33,31,34]$. This is true also for Pokémon GO, where social gaming motives have been found to correlate with derived health benefits, including physical activity and social well-being [35, 36, 37, 38].

\subsection{Other Location-based Health Apps}

Pokémon GO is by no means the only game in the market which has aimed to increase physical activity for players [39]. It is in fact not even the first location-based game to do so, as, for example, Ingress [40, 41, 42] and Zombies, Run! [43, 44] are its predecessors. From the two games, Ingress is more similar to Pokémon GO, as both games rely on a real world map based user interface and gameplay consists of navigating to PoIs [13, 45]. Zombies, Run!, however, creates gameplay based on the users' movement, primarily running, and the main feedback while playing is audio, highlighting the exergame design where running is not interrupted at any point by having to look at a smartphone screen [44]. Omitting visual feedback from the gameplay does have its perks when focusing on exercise, but the game can become less engaging as a result. In fact, a newer LBG zombie game, which does include audiovisual feedback, The Walking Dead: Our World, has recently bypassed Zombies, Run! in popularity [2]. It is worth noting that exergames can in theory be both engaging and also maintain effective physical exercises [46]. Even if an engaging exergame would not motivate towards short term effective physical activity, it can have longer lasting health benefits, especially for otherwise physically inactive individuals, as players will keep on playing the game longer[47].

In addition to smartphone games and apps, gamified exercise systems based on wearable technology have been developed [48, 49]. The benefit of these solutions is that they do not get in the way of exercise by requiring attention to a smartphone screen [27], instead, they provide an additional layer of gamification to the exercise to further boost motivation towards it [50]. However, critical studies on long-term effects the use of wearable technologies have on weight loss indicate that in fact, wearable technologies might have a slight negative effect or no effect at all on long term weight loss [51]. However, Pokémon GO has been found to have the potential of activating physically inactive individuals with no prior motivation to exercise [9, 47]. In addition to being playable on the smartphone, the game also provides wearable technology to support exercise and ensure that players do not have to look at the screen while walking, namely the Pokémon Go Plus wristband and the Pokéball plus [14].

\subsection{Motivational Factors in Pokémon GO}

The motivational reasons for playing Pokémon GO are complex $[5,52,53]$ and motivation and engagement in the game correlate with health benefits derived from playing [35]. Early studies identified catching Pokémon and completing the pokédex, (a library of found pokémon), to be more intrinsically motivating than battling elements [52], however, the game has changed a lot since then with many new features such as raid battles [30], trading and PvP being added [22]. Generally studies understand that as players enjoy playing Pokémon GO, and playing the game requires a great deal of exercise, therefore naturally the more engaged players are with the game, the more they will exercise [35].

Exergames can have physical, social and cognitive benefits [54], however these benefits never emerge unless players are engaged and committed to playing. In Pokémon GO, users derive gratification from, at least, being outdoors, challenge, competing, socializing, ease of use, game enjoyment and nostalgia [5]. Nostalgia and childhood dreams of becoming Pokémon trainers have been found to have played a big role, at least in the early moments after the launch of Pokémon GO, on its popularity [7]. After the initial hype died off, players who were motivated by in-game features kept on playing while those who were more concerned with the general game quality stopped playing. Interestingly, personality traits have not been found to affect whether player continues or stops playing Pokémon GO [1].

Pokémon GO design emphasizes social connectivity in multiple ways, rewarding players for interacting with each other [37, 38]. In addition, what brings Pokémon GO players together is their shared passion for the game [36]. The formed social networks help players sustain an interest in the game and can play a role in how enjoyable the game is $[55,56,34]$. Relationships formed with real 
people can serve as a motivator to go out and exercise [57] and the multiplayer features can be seen beneficial especially for long term engagement [58].

\subsection{Objectives and Research Problem}

Based on previous research, Pokémon GO seems a promising exergame, as it (1) Reaches demographics who would not otherwise engage with exercise [9], (2) Integrates social elements, story and other elements of gamification with exercise [5], (3) subtly encourages exercise, but does not force it [59] and (4) has other beneficial implications besides exercise, mainly increased social interaction $[37,38,60,35,36]$, increased knowledge of surrounding places [11] and increased navigational and cartographic skills [61]. As the previous research is multidisciplinary and sometimes cross-disciplinary, synthesizing results of previous studies can be difficult. Despite some efforts to bring studies on the effects playing Pokémon GO has on physical activity together [62], a clear quantitative picture is missing. To holistically study the effects Pokémon GO has on physical activity, the systematic literature research approach was chosen.

\section{Research Design}

To address the described problem, the following research question was formulated: "What are the short-term and long-term physical activity impacts of playing Pokémon GO?". In conducting the current systematic literature review, guidelines from PRISMA Statement were adopted [63]. Peer-reviewed empirical studies on the effects of playing Pokémon GO on the player's physical activity were looked for.

\subsection{Information Sources, Search and Study Selection}

Elsevier's Scopus research database was chosen as the main source of scientific articles for the current study. Despite reported biases towards natural sciences, biomedical research and engineering, Scopus remains one of the most widely used, versatile and respected research databases [64]. The database was searched on April 1st, 2019, using its own built-in search engine using the keyword: "Pokémon" AND "GO". All articles which included Pokémon GO either in the title, keywords or in the abstract were selected for more detailed inspection.

All found papers $(\mathrm{N}=279)$ were read by two authors independently, and categorized based on their theme. If authors disagreed on whether to include or exclude a study, a third author was brought in for council until a decision was made. At this stage, the following papers were excluded:

- Duplicates

- Short or poster papers

- Works in progress and studies which were not peer reviewed

- Letters and Editorials

- Studies which were not in English

- Studies which were not related to Pokémon GO

The resulting set of papers $(\mathrm{N}=151)$ were read by the authors, and studies on the effects playing Pokémon GO has on physical activity and/or exercise were chosen for the final selection $(\mathrm{N}=22)$. From these, conceptual studies and studies using grounded theory were excluded, thus 20 studies remained.

\subsection{Data Items}

The final selection of papers $(\mathrm{N}=20)$ were read carefully by two authors, and the following information was obtained:

1. Method of measuring physical activity/exercise including instruments and their validity.

2. Amount of participants

3. Span of the study

4. In which country or countries was the study carried out.

5. Results on physical activity including statistical significance if available

\subsection{Synthesis of Studies}

As the main gameplay in Pokémon GO consists of walking [9], measuring the change in the amount of daily steps taken for Pokémon GO players was natural. A model on physical activity and sedentary lifestyle by Tudor-Locke and Bassett [65] classifies below 5000 steps per day as sedentary lifestyle, 5000-7499 daily steps as typical lifestyle, 7500-9999 to likely include occupational exercise or physical activities and over 10000 daily steps means a person can be classified as physically active [65]. In order to understand the magnitude of the effect Pokémon GO has on physical activity, this model was used as baseline, assuming that an increase of over 1000 daily steps could be considered substantial. 
However, there were studies which did not measure the increase in the daily steps taken, in fact studies deployed a wide range of methods and points of view. This was a challenge in constructing the synthesis of the results. Previous similar studies have consorted to using qualitative summaries of results [62], which can be an issue when attempting to compare studies together. Some recent studies synthesizing quantitative results have done so by comparing outcomes with similar approaches together [66]. Therefore this study takes a similar approach by comparing studies using wearable devices for measurement together and comparing studies utilizing questionnaires (validated and non-validated) together in order to obtain a holistic picture of the effects Pokémon GO has had on players physical activity.

\section{Results}

After obtaining the studies from Scopus, altogether 279 studies were recorded. From those papers $44 \%$ were journal articles and $31 \%$ conference papers. The rest of the papers were categorized into e.g. book chapters, letters and editorials in Scopus's own characterization. The majority of the papers were from the fields of computer science, social sciences, medicine and engineering and almost one third of all papers was written by U.S.-based scholars. From all 279 studies, 142 papers were published in 2017, which roughly correlates with the launch of Pokémon GO in summer 2016.

\subsection{Descriptive statistics}

In the identification phase, depicted in Figure 2, duplicates were removed, after which 277 studies remained. Subsequently, in the screening phase, studies that did not meet the inclusion criteria defined in Section 3.1 were excluded. The remaining 151 studies were read in full and categorized according to their theme such as physical activity and exercise, cartography, geography and topography, LBG framework/taxonomy development $\&$ terminology, social interaction, law and lawsuits, economy and business, safety and security issues, player behavior and enjoyment and popularity of the game. Finally, 22 studies discussing the effects of Pokemon GO on physical activity and exercise remained. 21/22 of the studies were empirical and one was conceptual. However, out of the 21 papers, one utilized the grounded theory methodology in order to construct a theory concerning physical activity related issues in Pokémon GO [67]. As the paper differed from the others in chosen methods and focus, it was not included in the final synthesis, instead the results of the paper Yee et al. [67] are discussed separately in Section 4.3.

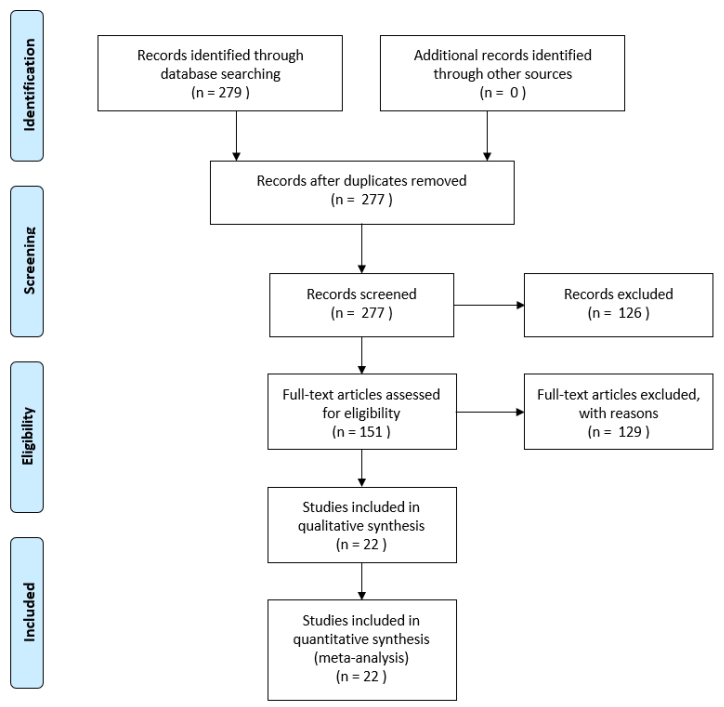

Figure 2. The systematic literature search process of Pokémon GO studies

\subsection{Study Characteristics}

The measurement methods, number of participants, span of the study and the country of participants of the 20 empirical studies are displayed in Table 1. Most studies used a questionnaire to collect data $(60 \%)$, with some sort of a wearable device collecting sensor data being the second most popular category $(25 \%)$. The three remaining papers collected data from multiple sources, including questionnaires, sensor data and interviews. Surprisingly, almost half (45\%) of all studies were conducted in the United States. Hong Kong was featured twice $(10 \%)$ and three other countries once $(15 \%)$. The rest of the studies were carried out globally. However, these were all online surveys, which depending on the selected method of distributing the survey, are prone to bias towards types of players and certain countries.

Most data in the studies shown in Table 1 was collected in 2016. However, as Pokémon GO has changed significantly since then [22], the results of the studies might partially be out of date. For example, in 2017 raids were introduced to the game, which added the social encouragement for players to travel to gyms and participate in collaborative raid battles to take down strong NPC foes [30]. The addition of cooperative elements is important as studies have shown they can influence the motivation for exercise [57]. 


\begin{tabular}{|c|c|c|c|c|c|c|c|}
\hline \multicolumn{8}{|c|}{ Synthesizes of Pokemon Go studies on physical activities } \\
\hline Paper & Study & Measuring method & $\mathbf{N}$ & $\begin{array}{l}\text { Data } \\
\text { year }\end{array}$ & Country & $\begin{array}{l}\text { Duration of } \\
\text { study }\end{array}$ & Change in physical activity \\
\hline 1 & Althoff et al., 2016 & $\begin{array}{ll}\text { Wearable } & \text { (Microsoft } \\
\text { Band) }\end{array}$ & $\begin{array}{l}31 \\
793\end{array}$ & 2016 & USA & 3 months & 1473 more steps \\
\hline 2 & Ni et al., (2019) & $\begin{array}{l}\text { Wearable (iPhone Health } \\
\text { App) }\end{array}$ & 65 & 2016 & China & 100 days & no change ( $1.5 \mathrm{~km}$ more walking at the peak) \\
\hline 3 & Xian et al., (2017) & $\begin{array}{l}\text { Wearable (iPhone Health } \\
\text { App) }\end{array}$ & 167 & 2016 & USA & 6 weeks & 1976 more steps \\
\hline 4 & Hino et al., (2019) & $\begin{array}{ll}\text { Wearable } & \text { (Free } \\
\text { pedometer) } & \end{array}$ & 230 & $2016-2017$ & Japan & 9 months & no change ( 368 more steps at peak) \\
\hline 5 & Marquet et al. (2018) & $\begin{array}{lr}\text { Wearable } & \text { (Ecological } \\
\text { Momentary } & \text { Assessment } \\
\text { (EMA) + IPAQ }\end{array}$ & 74 & 2016 & USA & 7 days & 1526 steps more for active players \\
\hline 6 & Howe et al., 2016 & Questionnaire (Own) & 1182 & 2016 & USA & 6 weeks & 955 more steps \\
\hline 7 & Wong, F.Y, (2017) & Questionnaire (IPAQ) & 644 & 2016 & China & Single survey & No change \\
\hline 8 & Kogan et al., (2017) & Questionnaire (Own) & 269 & 2016 & USA & Single survey & Self reported increase in exercise \\
\hline 9 & Liu \& Ligmann-Zielinska (2017) & Questionnaire (Own) & 47 & 2016 & USA & 7 days & Self reported increase in exercise \\
\hline 10 & Lalot et al., (2017) & $\begin{array}{l}\text { Questionnaire (Hexaco } 6 \\
\text { trait) }\end{array}$ & 402 & 2016 & $\begin{array}{l}10 \\
\text { countries }\end{array}$ & 5 months & $\begin{array}{l}1.56 \mathrm{~km} \text { walked daily average with Pokemon } \\
\text { GO. }\end{array}$ \\
\hline 12 & Gabbiadini (2018) & Questionnaire (Own) & 981 & N/A & Global & Single survey & $\begin{array}{l}\text { Physical activity reported to correlate with } \\
\text { playing }\end{array}$ \\
\hline 13 & Kaczmarek et al., (2017) & Questionnaire (IPAQ) & 444 & 2016 & Global & 6 weeks & $\begin{array}{l}\text { Physical activity reported to correlate with } \\
\text { playing }\end{array}$ \\
\hline 14 & Meschtscherjakov (2017) & Questionnaire (Own) & 124 & 2016 & Global & Single survey & Self reported increase in exercise \\
\hline 15 & Broom et al., (2018) & Questionnaire (IPAQ) & 461 & 2016 & Global & 3 months & Self reported increase in exercise \\
\hline 16 & Militello et al., (2018) & Questionnaire (Godin) & 160 & 2017 & USA & 2 months & Self reported increase in exercise \\
\hline 17 & Finco et al (2018) & Questionnaire (Own) & 125 & 2017 & Brazil & Single survey & $\begin{array}{l}\text { Informal learning happened on physical } \\
\text { activity }\end{array}$ \\
\hline 18 & Beach et al., (2019) & $\begin{array}{l}\text { Mixed (Pedometer + Own } \\
\text { surveys) }\end{array}$ & 100 & 2016 & USA & 12 days & Negative change in physical activity \\
\hline 19 & Barkley et al., (2017) & $\begin{array}{l}\text { Mixed (IPAQ }+ \text { Own } \\
\text { survey) }\end{array}$ & 358 & 2016 & USA & 3 weeks & no change \\
\hline 20 & Ma et al (2018) & $\begin{array}{l}\text { Mixed (Own survey }+ \\
\text { iPhone Health App) }\end{array}$ & 210 & 2016 & China & 35 days & No change (peak 1200 increase in steps) \\
\hline
\end{tabular}

Table 1. Synthesizes of Pokemon Go studies on physical activities

\subsection{Results of Individual Studies}

In the final set of studies, there were a few outliers. For example, Gabbiadini et al.[68] agree with many other studies that Pokémon GO improves players' physical activity. They argue, however, that these effects do not reliably encourage people to assume a more physically active lifestyle in general. Broom and Flint [59] on the other hand, observed that many players were able to replace Pokémon GO with some other type of physical activity after quitting playing. Bruno [12] notes that Pokémon GO can also be used in ways that do not involve players "wandering aimlessly with their eyes glued to the screen". For example at schools, Pokémon GO can be used imaginatively to promote fitness and physical activity. In this sense, Pokémon GO can also be used to spur physical activity not only motivated by the game itself. However, Yee et al.[67] had quite a different methodology from the other studies in this review, as two of the authors engaged in participant observations in using the technology with Pokémon GO players and collected data by taking field notes. Still, the result of the study is similar to many other papers, concluding that Pokémon GO can affect the lifestyle and activity of the players positively. Besides the above mentioned outliers, multiple studies highlighted that Pokémon GO has several ways for motivating players to exercise. These included gameplay and social factors [5].

\subsection{Synthesis of Studies}

The 20 empirical studies were observed for their reported change in physical activity and the duration of the study, and the results are shown in Table 1. In case at the end of a study there was no observable changes in physical activity, or the change was only brief, then the study was marked as "no change in physical activity.", however, this was likely because participants lost motivation to play. The increase in the amount of daily steps was reported from those studies which measured and reported the information. Those questionnaire studies which asked players to give their own impressions concerning a change in their physical activity, were marked as "self reported increase in physical activity". From the studies which analyzed correlation values between physical activity and game related events, the significance of the correlation between playing and physical activity was reported. Additionally, there was one study which reported an increase in the amount of meters walked per day. Finally, one study found Pokémon GO to have a negative impact on physical activity, and it was reported 
as such.

Six papers reported no change or only brief change, five papers reported a quantitatively measured increase in physical activity, five papers reported a self reported an increase in physical activity and two papers reported a significant correlation between playing Pokémon GO and increased physical activity. One paper from Beach et al. [69] reported that when out exercising for one hour, the exercise provided by Pokémon GO is not as effective as alternatives. Furthermore, study by Finco et al.[37] reported that Pokémon GO supports informal learning related physical activity.

Research by Hino et al. [17] carried out the longest study, lasting for a period of nine months and extending over winter, but the rest of the studies were shorter with an average duration of all empirical studies being 52 days. The most common time span of data collection was a single one time questionnaire, used by four studies. A 3-month time period was the second most popular. In addition, most data was collected in 2016, after which the game has changed a lot [22]. The findings highlight that studies on the long term effects (over a year) playing Pokémon GO has on physical activity are missing.

\subsection{Bias in the Observed Empirical Studies}

Many of the analyzed studies were based on questionnaires, and IPAQ [70] was the most commonly used validated instrument for measuring physical activity with the Godin [71] instrument being the second most popular. Questionnaires are perceived more prone to bias than quantitative data from wearable devices. For example, a positive conception of Pokémon GO, and time and money already invested in the game, might cause the participant to assess the frequency and the amount of physical activity too favorably. Also, the platforms and online communities which some studies used to recruit participants with, may have affected the results.

The studies having participants carry wearable sensors are more accurate in measuring exact data on how much the player's physical ability has increased. However, consciously carrying a sensor (e.g. a pedometer) may have an effect on the results, as the participant knows their performance is being tracked. Also as mentioned before, Pokémon GO has significantly changed and evolved as a game and the current version might have a different impact on players than the one used in the majority of studies. As Pokémon GO is a LBG and the playing location has an impact on the gameplay, the data collection location could have been specified. For a majority of the studies this was either not given in the text, i.e. did players play mainly in cities or rural environments.

\section{Discussion and Future Work}

\subsection{Key Findings}

The results reveal that playing Pokémon GO does increase mild physical activity. The studies which reported the effects the game has on daily number of steps taken, showed a daily increase ranging between 368-1976 steps. However, interestingly six studies found that the effects the game has on physical activity diminished quickly, and as soon as 6 weeks the amount of steps taken had returned to baseline. This apparent contradiction between studies which report a prolonged effect on physical activity and studies which report a quickly diminishing effect on physical activity could be solved by studies which observe the effects on physical activity in over the span of a year or more, however, currently such studies are missing.

Even though 20 empirical studies on the effects playing Pokémon GO has on physical activity were discovered, the used methods and focus of the studies varied quite a lot. This was a challenge for an accurate synthesis of the results, but also speaks for the complexity of Pokémon GO, its underlying complex motivational effects [5] and its novelty. Nonetheless, the results of the analyzed studies mostly followed along the same lines. Even the one paper which reported that playing Pokémon GO reduces physical activity [69], did so in a setting comparing physical activities with one another during a fixed time period. The results of the study [69], point towards the same conclusion as all the other evidence, that the type of exercise which Pokémon GO promotes is not intense, but light, fun and enjoyable. Perhaps the purpose of games such as Pokémon GO for public health is not to provide strenuous exercise, but to act as a gateway for physically inactive people to start exercising [9] and to provide friends and family a fun, social and healthy outdoor activity $[37,38]$.

\subsection{Limitations}

Even though Scopus is considered a high quality research database which includes research papers from the majority of major publications [64], there might have been some relevant studies which were not included in the current review. In addition, because Pokémon GO is still quite a new phenomenon, some research papers, especially those investigating the long-term effects the game has on exercise and physical activity, might still be in review or even not submitted, and thus, the data from those studies was also not included in the current 
study.

Besides the search, also the characterization and the synthesis of the studies had limitations. As various questionnaire instruments such as IPAQ [70] and Godin [71] were used, the results could perhaps not be compared with each other accurately. Additionally, the wearable devices used to measure physical activity had variance. The step counting measurement was used in the current study for estimating the level of physical activity, but even step counting algorithms have variance [72]. Furthermore, motivational impacts the game has on exercise were not included in the synthesis and were only discussed in light of literature.

In addition to the effects Pokémon GO has on physical activity, the game has several other health benefits which could not be included in the current study. Besides the much discussed increase in social interaction [30,38], the game can activate players and help with social withdrawal symptoms [73]. These benefits on players' health might in fact be more impactful than the effects the game has on physical activity, as they arguably last longer. The health benefits derived from Pokémon GO and exergames in general depend on that the games are being played. As Pokémon GO is constantly being updated and developed further [22], players retain their enthusiasm towards the game better, but this also means that studies on the impact of the game might become outdated.

\subsection{Future Work}

An interesting topic for further discussion is how Pokémon GO compares to other HBCSSs [16, 23] fitness apps and more importantly, other location-based games such as Ingress, The Walking Dead: Our World, Jurassic World: Alive and the upcoming Harry Potter: Wizards Unite. In addition, the positive effects Pokémon GO has on social well-being could be studied in further detail, as the game encourages talking to people face-to-face and being outdoors. The many instruments used for measuring physical activity (IPAQ, Godin, Hexaco 6 trait) suggest that a new instrument designed specifically for measuring physical activity in LBGs could be developed.

\section{Conclusions}

In this paper, the effects of playing the location-based game Pokémon GO on physical activity were studied. The current research on Pokémon GO was obtained via the systematic literature review approach. After screening the selection of papers, finally 20 empirical studies on the effects playing Pokémon GO has on physical activity remained. The results of the literature review showed that $30 \%$ of empirical studies on the effects Pokémon GO has on physical activity reported no increase in the long run, whereas $60 \%$ of papers reported an increase in mild physical activity, an increase of 500-1500 daily steps. In addition, the results show that the majority of data on the effects Pokémon GO has on physical activity was collected in 2016, back when the game was very different than today in 2019. In addition, longitudinal studies spanning for over a year are missing.

\section{References}

[1] P. Rasche, A. Schlomann, and A. Mertens, "Who is still playing pokemon go? a web-based survey," JMIR serious games, vol. 5, no. 2, p. e7, 2017.

[2] S. Laato., T. Pietarinen., S. Rauti., M. Paloheimo., N. Inaba., and E. Sutinen., "A review of location-based games: Do they all support exercise, social interaction and cartographical training?," in Proceedings of the 11th CSEDU - Volume 1: CSEDU, pp. 616-627, INSTICC, SciTePress, 2019.

[3] N. Liberati, "Phenomenology, pokémon go, and other augmented reality games," Human studies, vol. 41, no. 2 , pp. 211-232, 2018.

[4] A. McWhirter, "Pokémon go and no man's sky: sandbox gaming's next level in tech-heavy society," International Journal of Jungian Studies, vol. 9, no. 1, pp. 66-71, 2017.

[5] J. Hamari, A. Malik, J. Koski, and A. Johri, "Uses and gratifications of pokémon go: Why do people play mobile location-based augmented reality games?," International Journal of Human-Computer Interaction, vol. 35, no. 9, pp. 804-819, 2019.

[6] A. L. Rossow, "Gotta catch a lawsuit: A legal insight into the intellectual, civil, and criminal battlefield pokemon go has downloaded onto smartphones and properties around the world," J. Marshall Rev. Intell. Prop. L., vol. 16, p. i, 2016.

[7] A. K. Tang, "Key factors in the triumph of pokémon go," Business Horizons, vol. 60, no. 5, pp. 725-728, 2017.

[8] G. Goggin, "Locating mobile media audiences: In plain view with pokémon go," in Studying Digital Media Audiences, pp. 39-59, Routledge, 2017.

[9] T. Althoff, R. W. White, and E. Horvitz, "Influence of pokémon go on physical activity: study and implications," Journal of medical Internet research, vol. 18, no. 12, p. e315, 2016.

[10] C. R. Nigg, D. J. Mateo, and J. An, "Pokémon go may increase physical activity and decrease sedentary behaviors," American journal of public health, vol. 107, no. 1, p. 37, 2017.

[11] T. Oleksy and A. Wnuk, "Catch them all and increase your place attachment! the role of location-based augmented reality games in changing people-place relations," Computers in Human Behavior, vol. 76, pp. 3-8, 2017.

[12] L. E. Bruno, "Embracing technology and pop culture trends in physical education: Ready, set,(pokémon) go!," Journal of Physical Education, Recreation \& Dance, vol. 89, no. 4, pp. 45-51, 2018. 
[13] S. Laato., T. Pietarinen., S. Rauti., and T. H. Laine, "Analysis of the quality of points of interests in the most popular location-based games," in Proceedings of CompSysTech19, Sofia, Bulgaria, ACM, 2019.

[14] J. Sablatura and U. Karabiyik, "Pokémon go forensics: An android application analysis," Information, vol. 8, no. 3, p. 71, 2017.

[15] H. Oinas-Kukkonen, "Behavior change support systems: A research model and agenda," in International Conference on persuasive technology, pp. 4-14, Springer, 2010.

[16] H. Oinas-Kukkonen, "A foundation for the study of behavior change support systems," Personal and ubiquitous computing, vol. 17, no. 6, pp. 1223-1235, 2013.

[17] K. Hino, Y. Asami, and J. S. Lee, "Step counts of middle-aged and elderly adults for 10 months before and after the release of pokémon go in yokohama, japan," Journal of medical Internet research, vol. 21, no. 2, p. e10724, 2019.

[18] F. J. Zach and I. P. Tussyadiah, "To catch them all-the (un) intended consequences of pokémon go on mobility, consumption, and wellbeing," in Information and communication technologies in tourism 2017, pp. 217-227, Springer, 2017.

[19] L. Juhász and H. H. Hochmair, "Where to catch "em all?-a geographic analysis of pokémon go locations," Geo-spatial information science, vol. 20, no. 3, pp. 241-251, 2017.

[20] X. Tong, A. Gupta, H. Lo, A. Choo, D. Gromala, and C. D. Shaw, "Chasing lovely monsters in the wild, exploring players' motivation and play patterns of pokémon go: Go, gone or go away?," in Companion of the 2017 ACM CSCW, pp. 327-330, ACM, 2017.

[21] K. Sato, K. Kuroki, S. Saiki, and R. Nagatomi, "Improving walking, muscle strength, and balance in the elderly with an exergame using kinect: A randomized controlled trial," Games for health journal, vol. 4, no. 3, pp. 161-167, 2015.

[22] Niantic, "Pokémon go updates, https://pokemongolive.com/en/post/," 2019.

[23] P. Karppinen, H. Oinas-Kukkonen, T. Alahäivälä, T. Jokelainen, A.-M. Teeriniemi, T. Salonurmi, and M. J. Savolainen, "Opportunities and challenges of behavior change support systems for enhancing habit formation: A qualitative study," Journal of biomedical informatics, vol. 84, pp. 82-92, 2018.

[24] Y. Oh and S. Yang, "Defining exergames \& exergaming," Proceedings of Meaningful Play, pp. 1-17, 2010.

[25] C. Panumate, S. Xiong, H. Iida, and T. Kondo, "Evolutionary changes of pokemon game: A case study with focus on catching pokemon," in ICEC, pp. 182-194, Springer, 2015.

[26] C. McCrea, "We play in public: The nature and context of portable gaming systems," Convergence, vol. 17, no. 4, pp. 389-403, 2011.

[27] L. Chittaro and F. Zuliani, "Exploring audio storytelling in mobile exergames to affect the perception of physical exercise," in 2013 7th PervasiveHealth, pp. 1-8, IEEE, 2013.

[28] K. Moore, "Sort mii out: Learning to value portable gaming encounters through nintendo's streetpass software," Games and Culture, vol. 13, no. 6, pp. 587-604, 2018.
[29] S. Paasovaara and T. Olsson, "Proximity-based automatic exchange of data in mobile gaming: Studying the experiences of streetpass users," in Proceedings of the 9th NordiCHI, p. 26, ACM, 2016.

[30] A. Bhattacharya, T. W. Windleharth, R. A. Ishii, I. M. Acevedo, C. R. Aragon, J. A. Kientz, J. C. Yip, and J. H. Lee, "Group interactions in location-based gaming: A case study of raiding in pokémon go," in Proceedings of the 2019 CHI Conference on Human Factors in Computing Systems, p. 587, ACM, 2019.

[31] W. Peng and J. Crouse, "Playing in parallel: The effects of multiplayer modes in active video game on motivation and physical exertion," Cyberpsychology, behavior, and social networking, vol. 16, no. 6, pp. 423-427, 2013.

[32] K. Vered, Children and media outside the home: playing and learning in after-school care. Springer, 2008.

[33] S. Göbel, S. Hardy, V. Wendel, F. Mehm, and R. Steinmetz, "Serious games for health: personalized exergames," in Proceedings of the 18th ACM international conference on Multimedia, pp. 1663-1666, ACM, 2010.

[34] Y. Xu, E. S. Poole, A. D. Miller, E. Eiriksdottir, D. Kestranek, R. Catrambone, and E. D. Mynatt, "This is not a one-horse race: understanding player types in multiplayer pervasive health games for youth," in Proceedings of the ACM 2012 CSCW, pp. 843-852, ACM, 2012.

[35] L. D. Kaczmarek, M. Misiak, M. Behnke, M. Dziekan, and P. Guzik, "The pikachu effect: Social and health gaming motivations lead to greater benefits of pokémon go use," Computers in Human Behavior, vol. 75, pp. 356-363, 2017.

[36] K. Vella, D. Johnson, V. W. S. Cheng, T. Davenport, J. Mitchell, M. Klarkowski, and C. Phillips, "A sense of belonging: Pokemon go and social connectedness," Games and Culture, p. 1555412017719973, 2017.

[37] M. D. Finco, R. S. Rocha, R. W. Fão, and F. Santos, "Let's move!: The social and health contributions from pokémon go," International Journal of Game-Based Learning (IJGBL), vol. 8, no. 2, pp. 44-54, 2018.

[38] M. D. Finco, "I play, you play and we play together: Social interaction through the use of pokémon go," in Augmented Reality Games I, pp. 117-128, Springer, 2019.

[39] A. Matallaoui, J. Koivisto, J. Hamari, and R. Zarnekow, "How effective is "exergamification"? a systematic review on the effectiveness of gamification features in exergames," in Proceedings of the 50th HICSSH, 2017.

[40] M. Davis, "Ingress in geography: Portals to academic success?," Journal of Geography, vol. 116, no. 2, pp. 89-97, 2017.

[41] M. Majorek and M. Du Vall, "Ingress: an example of a new dimension in entertainment," Games and Culture, vol. 11, no. 7-8, pp. 667-689, 2016.

[42] H. Söbke, J. B. Hauge, and I. A. Stefan, "Prime example ingress reframing the pervasive game design framework (pgdf)," International Journal of Serious Games, vol. 4, no. $2,2017$.

[43] C. Southerton, "Zombies, run! rethinking immersion in light of nontraditional gaming contexts," in Transmedia Practice: A Collective Approach, pp. 131-141, Brill, 2014. 
[44] E. Witkowski, "Running with zombies: Capturing new worlds through movement and visibility practices with zombies, run!," Games and Culture, vol. 13, no. 2, pp. 153-173, 2018.

[45] T. Tregel, L. Raymann, S. Göbel, and R. Steinmetz, "Geodata classification for automatic content creation in location-based games," in Joint International Conference on Serious Games, pp. 212-223, Springer, 2017.

[46] J. Höysniemi, A. Aula, P. Auvinen, J. Hännikäinen, and P. Hämäläinen, "Shadow boxer: a physically interactive fitness game," in Proceedings of the third NordCHI, pp. 389-392, ACM, 2004.

[47] A. G. LeBlanc and J.-P. Chaput, "Pokémon go: A game changer for the physical inactivity crisis?," Preventive medicine, vol. 101, pp. 235-237, 2017.

[48] R. Lindberg, J. Seo, and T. H. Laine, "Enhancing physical education with exergames and wearable technology," IEEE TLT, vol. 9, no. 4, pp. 328-341, 2016.

[49] Z. Zhao, S. A. Etemad, A. Whitehead, and A. Arya, "Motivational impacts and sustainability analysis of a wearable-based gamified exercise and fitness system," in Proceedings of the 2016 CHI PLAY, pp. 359-365, ACM, 2016.

[50] D. Johnson, S. Deterding, K.-A. Kuhn, A. Staneva, S. Stoyanov, and L. Hides, "Gamification for health and wellbeing: A systematic review of the literature," Internet interventions, vol. 6, pp. 89-106, 2016.

[51] J. M. Jakicic, K. K. Davis, R. J. Rogers, W. C. King, M. D. Marcus, D. Helsel, A. D. Rickman, A. S Wahed, and S. H. Belle, "Effect of wearable technology combined with a lifestyle intervention on long-term weight loss: the idea randomized clinical trial," Jama, vol. 316, no. 11, pp. 1161-1171, 2016.

[52] A. Meschtscherjakov, S. Trösterer, A. Lupp, and M. Tscheligi, "Pokémon walk: Persuasive effects of pokémon go game-design elements," in PERSUASIVE 2017, pp. 241-252, Springer, 2017.

[53] M. E. Tabacchi, B. Caci, M. Cardaci, and V. Perticone, "Early usage of pokémon go and its personality correlates," Computers in Human Behavior, vol. 72, pp. 163-169, 2017.

[54] A. E. Staiano and S. L. Calvert, "Exergames for physical education courses: Physical, social, and cognitive benefits," Child development perspectives, vol. 5 , no. 2 , pp. 93-98, 2011.

[55] H. Cole and M. D. Griffiths, "Social interactions in massively multiplayer online role-playing gamers," Cyberpsychology \& behavior, vol. 10, no. 4, pp. 575-583, 2007.

[56] B. Morschheuser, M. Riar, J. Hamari, and A. Maedche, "How games induce cooperation? a study on the relationship between game features and we-intentions in an augmented reality game," Computers in human behavior, vol. 77, pp. 169-183, 2017.

[57] J. Hamari and J. Koivisto, “"wworking out for likes”: An empirical study on social influence in exercise gamification," Computers in Human Behavior, vol. 50, pp. 333-347, 2015.

[58] K. Park, M. Cha, H. Kwak, and K.-T. Chen, "Achievement and friends: Key factors of player retention vary across player levels in online multiplayer games," in Proceedings of the 26th WWW2017, pp. 445-453, WWW2017, 2017.
[59] D. R. Broom and S. W. Flint, "Gotta catch "em all: Impact of pokémon go on physical activity, sitting time, and perceptions of physical activity and health at baseline and three-month follow-up," Games for health journal, vol. 7, no. 6, pp. 401-408, 2018.

[60] L. Humphreys, "Involvement shield or social catalyst: Thoughts on sociospatial practice of pokémon go," Mobile Media \& Communication, vol. 5, no. 1, pp. 15-19, 2017.

[61] C. Carbonell Carrera, J. L. Saorín, and S. Hess Medler, "Pokémon go and improvement in spatial orientation skills," Journal of Geography, vol. 117, no. 6, pp. 245-253, 2018.

[62] T. Baranowski and E. J. Lyons, "Scoping review of pokémon go: Comprehensive assessment of augmented reality for physical activity change," Games for health journal, 2019.

[63] D. Moher, A. Liberati, J. Tetzlaff, and D. G. Altman, "Preferred reporting items for systematic reviews and meta-analyses: the prisma statement," Annals of internal medicine, vol. 151, no. 4, pp. 264-269, 2009.

[64] P. Mongeon and A. Paul-Hus, "The journal coverage of web of science and scopus: a comparative analysis," Scientometrics, vol. 106, no. 1, pp. 213-228, 2016.

[65] C. Tudor-Locke and D. R. Bassett, "How many steps/day are enough?," Sports medicine, vol. 34, no. 1, pp. 1-8, 2004.

[66] A. Baye, A. Inns, C. Lake, and R. E. Slavin, "A synthesis of quantitative research on reading programs for secondary students," Reading Research Quarterly, vol. 54, no. 2, pp. 133-166, 2019.

[67] K. C. Yee, M. C. Wong, and P. Turner, "Pokemon go: ubiquitous computing delivering better health or co-incidential health benefits from technology use? a participatory observational study," Studies in health technology and informatics, vol. 234, no. 1, pp. 389-394, 2017.

[68] A. Gabbiadini, C. Sagioglou, and T. Greitemeyer, "Does pokémon go lead to a more physically active life style?," Computers in Human Behavior, vol. 84, pp. 258-263, 2018.

[69] C. Beach, G. Billstrom, E. T. Anderson Steeves, J. I. Flynn, and J. A. Steeves, "The physical activity patterns of greenway users playing pokemon go: A natural experiment," Games for health journal, vol. 8, no. 1, pp. 7-14, 2019.

[70] M. Hagströmer, P. Oja, and M. Sjöström, “The international physical activity questionnaire (ipaq): a study of concurrent and construct validity," Public health nutrition, vol. 9, no. 6, pp. 755-762, 2006.

[71] G. Godin, "The godin-shephard leisure-time physical activity questionnaire," The Health \& Fitness Journal of Canada, vol. 4, no. 1, pp. 18-22, 2011.

[72] J. Seo and T. H. Laine, "Accurate position and orientation independent step counting algorithm for smartphones," Journal of Ambient Intelligence and Smart Environments, vol. 10, no. 6, pp. 481-495, 2018.

[73] M. Tateno, N. Skokauskas, T. A. Kato, A. R. Teo, and A. P. Guerrero, "New game software (pokémon go) may help youth with severe social withdrawal, hikikomori," Psychiatry research, vol. 246, p. 848, 2016. 